\title{
Enabling Trustworthiness in Artificial Intelligence - A Detailed Discussion
}

\author{
Siddhartha Vadlamudi \\ Quixey Inc., Vintech Solutions, USA \\ *Corresponding Contact: \\ vadlamudisiddhartha@gmail.com
}

\begin{abstract}
Artificial intelligence (AI) delivers numerous chances to add to the prosperity of people and the stability of economies and society, yet besides, it adds up a variety of novel moral, legal, social, and innovative difficulties. Trustworthy AI (TAI) bases on the possibility that trust builds the establishment of various societies, economies, and sustainable turn of events, and that people, organizations, and societies can along these lines just at any point understand the maximum capacity of AI, if trust can be set up in its development, deployment, and use. The risks of unintended and negative outcomes related to AI are proportionately high, particularly at scale. Most AI is really artificial narrow intelligence, intended to achieve a specific task on previously curated information from a certain source. Since most AI models expand on correlations, predictions could fail to sum up to various populations or settings and might fuel existing disparities and biases. As the $\mathrm{AI}$ industry is amazingly imbalanced, and experts are as of now overpowered by other digital devices, there could be a little capacity to catch blunders. With this article, we aim to present the idea of TAI and its five essential standards (1) usefulness, (2) non-maleficence, (3) autonomy, (4) justice, and (5) logic. We further draw on these five standards to build up a data-driven analysis for TAI and present its application by portraying productive paths for future research, especially as to the distributed ledger technology-based acknowledgment of TAI.
\end{abstract}

Key words

Trustworthiness, Artificial, Intelligence, Trust-worthy, AI

\section{INTRODUCTION}

Artificial intelligence (AI) empowers computers to execute tasks that are simple for individuals to perform however hard to describe formally. It is perhaps the most-discussed innovation pattern in research and practice today. Even though AI has been near and investigated for quite a long time, it is particularly the new advances in the subfields of 
machine learning and deep learning that not just outcome in complex freedoms to add to the prosperity of people as well as the development and advancement of organizations and societies, however, additionally a variety other challenges that may seriously hinder AI's worth commitments, if not took care of properly.

To expand the advantages of AI while simultaneously moderating or in any event, forestalling its risks and dangers, the idea of trustworthy AI (TAI) advances that people, associations, and societies can accomplish the maximum capacity of AI if trust can be set up in its development, deployment, and use.

Be that as it may, the significance of TAI isn't restricted to domains like medical services or autonomous driving yet stretches out to different areas too. Electronic industry sectors, for instance, are progressively expanded with AI-based frame-works, for example, customer support chatbots. Similarly, a few cloud providers as of late started offering 'AI as a Service' (AIaaS), referring to web services for associations and people keen on training, building, and deploying AI-based frameworks. In this paper, I will contend that simplicity and explain ability are fundamental to amplifying the probability that AI affects mankind. I articulate the significance of these highlights with regards to a joint effort among people and AI, communicating that it is important that we emphasize awareness and trustworthiness through significant simplicity and explain ability. Implying that AI can sufficiently communicate to people the process by which it makes judgments that will encourage dependence; this clarification is vital to a profitable connection among AI and people. Furthermore, I recommend that state guidelines will be vital for steer AI toward valuable closures and to monitor, prevent, and rectify different maltreatments. Communist standards appear most appropriate to build up and uphold highlights of simplicity and explain ability in AI. Eventually, it is significant that we develop and deploy AI frameworks thoughtfully, carefully seeking advancement to the degree that AI can be utilized as a tool that upgrades human profitability and allows humankind to prosper.

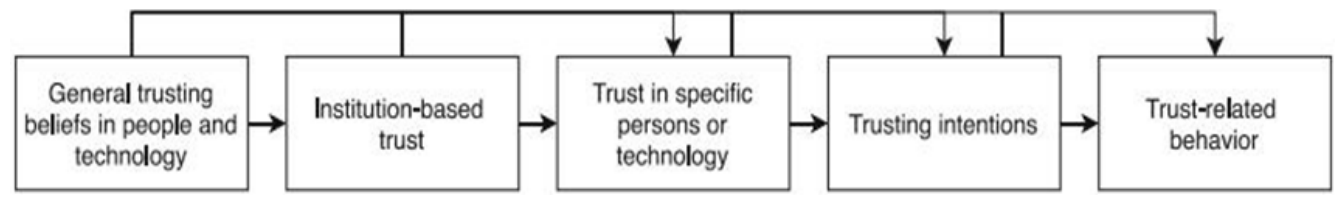

Fig. 1. Simplified Model of Trust in AI

\section{LITERATURE REVIEW}

AI can be characterized from multiple points of view-for the motivations behind this paper, it refers basically to machine learning (ML) algorithms that develop over time without being expressly programmed [1]. Algorithms are a bunch of rules to be followed; on account of ML, programming algorithms are trained with informational data sources and direction from people in regards to wanted results. In this paper, utilization of the expression "algorithms" refers to ML algorithms. These algorithms aim to enhance for a given measurement-regardless of whether it be a more proficient utilization of agricultural assets like water on a ranch that utilizes AI to monitor crop and yield, a more exact forecast of songs that may line up with your music taste when playing music with Spotify, or a viable assurance of what content would be generally engaging on Facebook to maximize clicks and revenue generation [2]. 
The scope of this paper includes weak or narrow AI-an implementation of AI innovation that is centered on a particular assignment or set of tasks. The restrictions of this classification of projects are not handily decided; narrow AI is best perceived with solid AI or artificial general intelligence (AGI), characterized as "machine intelligence with the full scope of human intelligence" [3]. Furthermore, functional implementations of AI today are best considered weak [4]. While this differentiation could get insignificant after the appearance of AGI, I note it to explain my argument, indicating that I am talking about present innovation and not-so-distant future pathways as opposed to what right now may be all the more precisely thought about sci-fi. All through this paper, I talk about AI as a tool that is educated by information and that is utilized by people. It is characteristic for AI that it captures human and historical inclination-once in a while apparently more objective, algorithms are not independent of those creating, deploying, and using them.

\section{INTERACTING WITH ARTIFICIAL INTELLIGENCE IN OUR DAILY LIFE}

Before we dive deeper into what risks and dangers lie ahead for AI, we must get to know what it's like to interact with $\mathrm{AI}$ in the first place to capture the shortcomings and hindrances it may present. Interacting with artificial intelligence or bots specifically is labeled as Conversational Artificial Intelligence. Conversational artificial intelligence (AI) refers to methodologies, like chatbots or voice assistants, with which users can converse. They use huge volumes of data, machine learning, and natural language processing to help emulate human interactions, recognizing speech and text inputs and interpreting their implications across different languages. These days, Artificial Intelligence (AI) is omnipresent. We can hardly open a newspaper or check out the news online without getting some story about AI. AI is presumably the innovation most discussed. Yet, AI implies various things to various individuals. Responsibility in AI starts with a legitimate AI narrative, which reveals the prospects and the cycles of AI technology and empowers that all can take an interest in the conversation on the part of AI in society. In this piece, we will attempt to explain what $\mathrm{AI}$ is, beginning by depicting how and what it is like to talk or interact with $\mathrm{AI}$ in our everyday life.

\section{What it's like to interact with AI?}

When people think of conversational artificial intelligence, online chatbots and voice assistants regularly strike a chord for their customer support administrations. Most conversational AI applications have broad analysis and data built into the back-end program, ensuring a human-like interaction experience. Analysts consider this sort of AI's present applications weak at this point, as they are centered on performing a limited amount of tasks. Strong AI, which is still a hypothetical idea, centers on a human-like consciousness that can make wiser decisions and perform a variety of different tasks and take care of a wide scope of issues. Regardless of its restricted focus, interacting with AI is an amazingly worthwhile innovation for tasks, helping organizations to be more productive and beneficial. While an AI chatbot is the most well-known type of talking with AI, there are numerous other use cases across the enterprise. Like healthcare services, HR processes, IoTbased devices, and that's just the beginning.

To the extent of one's daily communications with artificial intelligence, you get a front seat to experience that each time you speak to a virtual assistant like Siri, Alexa, or Bixby. At whatever point you ask your smartphone for directions, request paper towels from a virtual 
assistant, or give any non-human entity an order, you're speaking to a gadget controlled by AI. Virtual assistants use Natural Language Processing (NLP) to comprehend what you say to then give a response to your query. Social media is another platform that benefits from the ever-growing brain of artificial intelligence - Twitter as of late brought Watson, IBM's AI machine, on board to help prevent abuse by tracking problematic accounts.

\section{Features of Interacting with Artificial Intelligence (AI)}

Talking/interacting with AI combines natural language processing (NLP) with machine learning (ML). These NLP measures stream into a steady input cycle with machine learning cycles to constantly improve the AI calculations. AI has principle components that allow it to measure, understand, and create a response characteristically. This combination of NLP and ML comprises four features: Input generation, input analysis, output generation, and reinforcement learning. Unstructured data is transformed into a format that can be understood by a computer, which is then analyzed to create an appropriate response. Basic ML calculations improve response quality over the long haul as it learns. These four features can be separated further below:

- Input generation: Users provide input through a website or an application; the format of the data can either be voice or text.

- Input analysis: If the information is text-based, the conversational AI application will use natural language understanding (NLU) to understand the significance of the input and infer its goal. Be that as it may, if the information is voice-based, it'll influence a mix of automatic speech recognition (ASR) and NLU to analyze the information.

- Output generation: During this stage, Natural Language Generation (NLG), a part of NLP, forms a response.

- Reinforcement learning: Finally, machine learning algorithms refine the generated response over the long haul to ensure accuracy.

\section{How can you determine if it's a bot?}

When we chat with individuals on the Internet, we have the right to know if they are actual humans or we're just conversing with another AI bot. In a time where bots drive the majority of the web traffic, it's sensible for purchasers to be careful about chatbots masquerading people. A variety of bots talk with you on websites, for example, Facebook or some other ecommerce website. Programmers design chatbots to reproduce human-like interaction to persuade you to purchase something, click on a link, or offer individual data. The way to identifying them is by seeing how they work in different settings. At that point, you can exploit their shortcomings and out them as AI bots. So, here you go!

- $\quad$ Responds Suspiciously Quickly: Real human beings need to rest and take more than 0.1 seconds to type a detailed message. They will not react immediately and of course not at all the hours of the night.

- Doesn't Speak Naturally: The vast majority of people don't chat with a higher level of clarity and quickness. Real people use loads of sentence fragments when they're communicating. 
- Rehashed Answers: When individuals chat with bots, they are punching information into an if-then of programmed code. There's just such a lot of time to code, so a few responses may have more than one trigger. A human would not react to different questions or comments in the same manner.

- Strange Syntax: Here and there the way a bot produces text reflects mistakes in its programming. It tends to be something like two spaces between each sentence, additional full stops, or odd spaces.

As talked earlier, $\mathrm{AI}$ is evolving each day and trying to be more progressive and productive. The mentioned shortcomings might be resolved as time passes but AI is bound to leave its traces at some point and we - humans - will surely catch on to that as well.

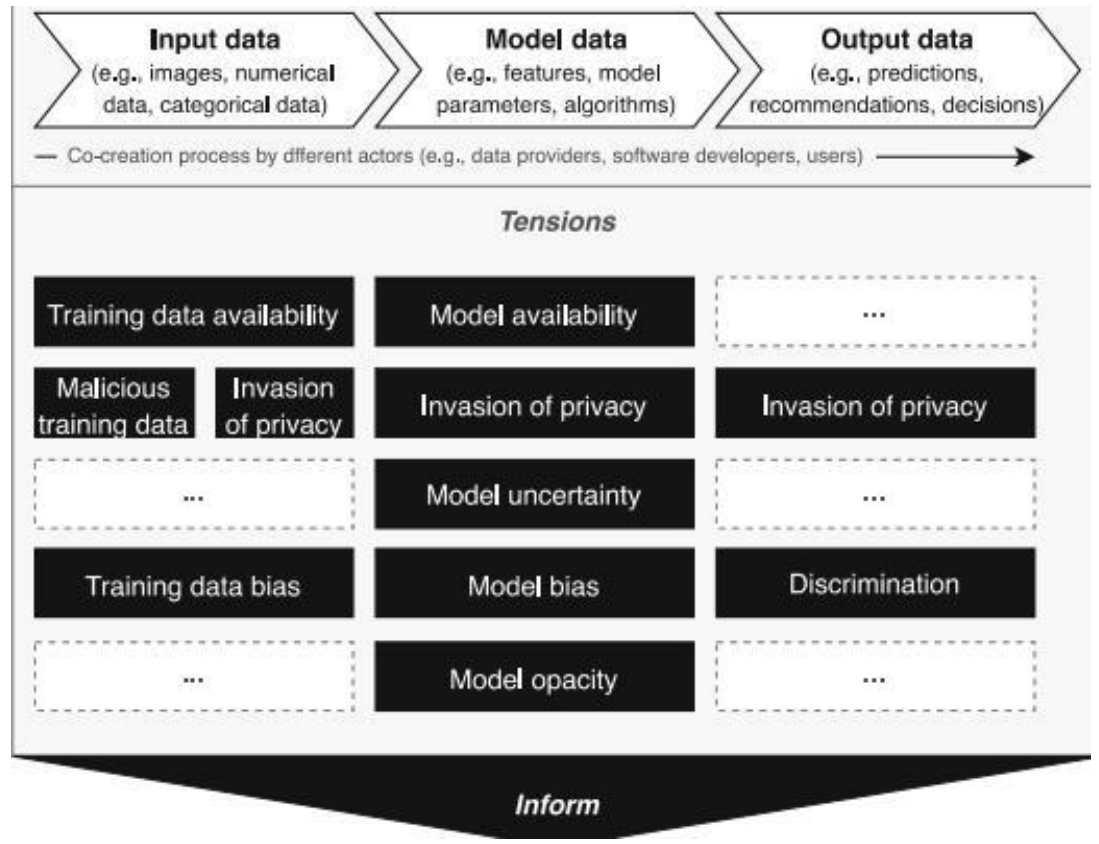

Fig. 2. Data as a key resource for AI-based systems

\section{TRUSTWORTHY ARTIFICIAL INTELLIGENCE}

This section is going to be the most important and detailed comprehension of what trustworthiness in AI systems should be like and how we can classify it.

A few systems and rules that promote moral standards for TAI have been created and distributed by analysts, industry, and policymakers in the past. Specifically, we adopt the five standards of moral AI; usefulness, non-maleficence, autonomy, justice, and logic which must be satisfied by an AI-based framework to be seen as trustworthy. These five standards combine different appropriate systems and rules as well as especially pertinent frameworks for digital business sectors since they reflect a socio-specialized view, underlining the connection among individuals and innovation that is expected to acknowledge TAI. 


\section{TAI PRINCIPLE}

\section{Usefulness}

Usefulness alludes to the development, organization, and utilization of AI that is valuable to humankind and the world as it advances the prosperity of people and the environment and regards fundamental basic liberties. Even though usefulness is found altogether of the systems and rules talked about here, it is considered to various degrees. While, for instance, a portion of the proposed frameworks and rules center this rule on the prosperity of humankind, others extend it to every conscious being and surprisingly the environment. The research identified with the usefulness principle, for the most part, comes from the areas of ethical computing and AI ethics, which center on talking about essential ethical topics and how to embed values that develop prosperity into AI at the design and development stages. From a wide point of view, the usefulness principle requests associations to consider, for instance, the environment (e.g., being sustainable and harmless to the ecosystem when using computing resources to deploy AI) as well as the cultural effect of AI services and products offered (e.g., installing AI-based chatbots that support buyers rather than just gathering further customer information).

\section{Non-maleficence}

Non-maleficence supports the development, deployment, and utilization of AI to such an extent that it tries not to carry a threat to individuals. Albeit like usefulness, which underscores the formation of AI that effectively acts towards the prosperity of humankind, non-maleficence addresses a particular principle that addresses a critical part of every thought about framework and principles. Non-maleficence particularly concerns the insurance of individuals' protection and security as well as their wellbeing. A fascinating feature of this current guideline's viewpoint along these lines rotates around artificial general intelligence (i.e., computer programs that can handle themselves and tackle difficulties in a wide range of domains) and how we can guarantee that artificial general intelligence, when it turns into a reality, acts in a non-destructive way.

Non-maleficence identifies with the confiding in convictions trustworthiness, dependability, and process since it requires AI-based frameworks to act genuinely and reliably and to truly hold fast to ethics and other pre-characterized standards. The nonmaleficence principle is exceptionally significant for digital business sectors because of the trade and analysis of profoundly sensitive buyers and licensed innovation information. For instance, organizations offering AIaaS should carry out sufficient information governance and assurance tools to such an extent that collected as well as AI-created data about people isn't utilized in a manner that obstructs their security and that users are empowered to more readily comprehend the results of data disclosure.

\section{Autonomy}

Autonomy is the third TAI principle. Given that surviving TAI frameworks and principles give marginally extraordinary understandings of this principle, it comes up short on an exact definition. While some mainly center on the advancement of human independence, agency, and oversight, others likewise consider the limitation of AI-based frameworks' selfgovernance, where vital. Specialists allude to this as meta-autonomy and people retaining the option to choose when to decide at some random time. Just two rules don't 
straightforwardly address the requirement for autonomy, The Chinese AI Principles conceptually refer to the requirement for 'controllability', expressing that "controllability of AI frameworks ought to be improved persistently" however don't further talk about their comprehension of this idea. Likewise, the White House AI Principles use autonomy to inspire a few different standards, expressing that AI may obstruct or add to human independence, be that as it may, don't refer to independence as a critical guideline in itself.

Examination on AI autonomy is assorted and includes, for instance, the autonomy of human-robot interactions as discussed in the 4th point, or the interaction of a few independent experts. Of specific worry according to this standard is research on trust in autonomous frameworks like autonomous vehicles, as well as research on adjustable autonomy, which refers to specialists powerfully changing their autonomy and moving it to different elements. For organizations, this principle infers that they ought to consider carrying out legitimate oversight measures (e.g., keeping the human-tuned in) to guarantee autonomy while installing AI into their electronic services and devices.

\section{Justice}

Like non-maleficence, justice is a key part of the trustworthy framework and principles examined in this article, but it is additionally referred to as fairness by a few. Justice isn't to be seen judicially, as in clinging to laws and guidelines, yet rather morally. All things considered, all frameworks and principles display comparable yet marginally particular perspectives on justice, which can be summed up as (1) the use of AI to correct past disparities like segregation, (2) the production of shareable and resulting conveyance of advantages through AI, and (3) impeding the making of new damages and imbalances by AI. Concerning the use of AI to revise past imbalances, for instance, agencies ought to consider "regardless of whether the AI application at issue may diminish levels of unlawful, unfair, or in any case, unintended separation when contrasted with existing processes and measures".

Justice in its different forms is a significant part of con-temporary AI research. Central research subjects concerning the justice standard are, for example, identifying the presence of racial and different inclinations in current AI-based frame-works, implies for measuring the fairness or absence thereof in AI-based frameworks, and approaches for mitigating or in any event, maintaining a strategic distance from bias in AI-based frameworks. Nevertheless, the justice rule is likewise exceptionally significant for electronic business sectors as, for instance, AI-based product suggestions might be affected by popularity biases, where popular items would be introduced more to the general population, while such a proposal may not be an aftereffect of good quality.

\section{Logic}

Logic is the fifth and last TAI rule. Logic involves an epistemological sense as well as a moral sense. In its epistemo-logical sense, logic entails the formation of explainable AI by delivering (more) interpretable AI models while maintaining significant degrees of execution and precision. In its moral sense, logic involves the formation of responsible AI. Inside the key structures and principles considered in this work, logic can be found under various terms and to fluctuating degrees. Experts pass on this standard by forming the requirement for simple $\mathrm{AI}$ and understandability of $\mathrm{AI}$, individually. 
Logic, in its two implications, is maybe the most predominant topic in contemporary AI research. A central justification lies in the way that the present AI-based frameworks are mind-boggling frameworks that generally work as secret elements and hence experience the ill effects of opacity and an absence of responsibility. Their portrayal of the state is regularly difficult to reach and complex to people, in this way restricting people from completely understanding and confiding in the different outputs. Logic is viewed as an empowering guideline for TAI, as it increases the four recently examined standards. Toward this end, "[One] should have the option to comprehend the great or damage [AI] is doing to society, and in which ways" for it to be beneficial and non-maleficent.

\section{Challenges in Fostering Responsibility, Trust, \& Trustworthiness in Al}

Comprehensively talking, responsibility is the establishment of trust in society. Responsibility is about a reasonable affirmation and acceptance of accountability and "answerability" for activities, choices, products, and approaches. Right now, three "meanings" of responsibility identified with AI exist in the literature, each highlighting an alternate locus for a certain activity. In the first meaning, responsibility is an element of the AI framework itself. [5] Building explain ability into the AI frameworks would incompletely address the AI's responsibility in this sense. The second meaning of responsibility centers on figuring out which people or teams are responsible for the effect of these algorithms or AI itself.

In this sense, responsibility is fairly barely connected with identifying who is generally liable for what impact inside the sociotechnical framework. At last, and maybe most comprehensively, responsibility is viewed as a component of the more extensive sociotechnical framework that creates, obtains, deploys, and utilizes AI. [7] For instance, AI Now proposes an Algorithmic Impact Assessment structure (like a Privacy Impact Assessment) as a method for incorporating responsibility into the more extensive sociotechnical frame-work in which AI is deployed, only part of which would have responsible judgments.

Another challenge for trustworthy $\mathrm{AI}$ is that $\mathrm{AI}$ is portable across various fields all over the world. It is developed and deployed in different locales, and in manners that cross worldwide and social limits. Delivery and development of advanced resources are hard to constrain. This entangles trust, for instance, when AI that is developed with one bunch of social suspicions implanted into it, is delivered in an "unfamiliar" social setting, where trust-building standards vary. It likewise misperceives individual jurisdictional responses, since an AI may or probably won't be worked to respect the local laws and fitting social standards. The challenges of managing cross-jurisdictional issues are not new, describing various issues in the digital age, protection being top among them. As we have seen with the new cross-jurisdictional arrangements require multi-stakeholder input and would profit by multi-horizontal coordination. This coordination couldn't just ensure that an AI is working inside the lawful constraints of various locales, but also that it is working securely and in a trustworthy way.

\section{CONCLUSION}

In this paper, I presented the idea of TAI as a promising research subject for research, depicted its experience, situated it in related trust conceptualizations, and contextualized the five TAI principles; usefulness, non-maleficence, autonomy, justice, and logic. Further, I drew on the challenges identified with responsibility, trust, and trustworthiness to build 
up the agreement that gives direction to those captivated to examine specialized and nonspecialized methods on the side of TAI, and presented its achievability for future research on TAI.

In doing as such, I feature a tremendous space of TAI research opportunities for the other researchers that aren't restricted to the new AI hype subject of explain ability. Particularly for the field of electronic business sectors, TAI gives a few promising options for future research, including and beyond its data-driven methodology. The strains between information at the various phases of the AI co-creation measure and the five TAI principles that we illustrated here address just a subset of challenges. All things considered, I am persuaded that these principles give a decent starting ground to exploring further strains and, hence, discovering more ways for future research on specialized and non-specialized methods on the side of TAI.

\section{REFERENCES}

[1] Acemoglu, Daron, and Pascual Restrepo, "Artificial Intelligence, Automation and Work," MIT Economics, January 4, 2018, https:/ /economics.mit.edu/files/14641.

[2] Akata, Zeynep, Trevor Darrell, Lisa Anne Hendricks, Dong Huk Park, Marcus Rohrbach, and Bernt Schiele, "Attentive Explanations: Justifying Decisions and Pointing to the Evidence." ARXIV, December 2016. https:/ / arxiv.org/pdf/1612.04757v1.pdf.

[3] Angwin, Julia, Jeff Larson, Surya Mattu, and Lauren Kirchner. "Machine Bias." ProPublica, 23 May 2016. https://www.propublica.org/article/machine-bias-risk-assessments-incriminalsentencing.

[4] Armstrong, Stuart. "AI safety: three human problems and one AI issue." Intelligent Agent Foundations Forum, May 19, 2017. https:/ /agentfoundations.org/item?id=1388.

[5] Doshi-Velez Kortz; Villani, C. (2018). “For a Meaningful Artificial In-telligence: Towards A French and European Strategy." Ai for Humanity. Online: https:/ /www.aiforhumanity.fr/pdfs/MissionVillani-Report-ENG-VF.pdf

[6] House of Lords, Select Committee on Artificial Intelligence. (2018). "AI in the UK: ready, willing and able?", Yeung, K. quoted at $96 . \quad$ Online: https://publications.parliament.uk/pa/ld201719/ldselect/ldai/100/100.pdf; Larus, J. et al. (2018). "When Computers Decide: European Recommendations on Machine-Learned Automated Decision Making." Informatics Europe EUACM. Online: http://w ww.informaticseurope.org/component/phocadow nload/category/10- reports.html?dow nload=74:automateddecision-making-report

[7] Reisman, D. et al. (2018); House of Lords, 35. 
Online Archive Link: https://abc.us.org/ojs/index.php/ei/issue/archive 